Elect. Comm. in Probab. 12 (2007), 140-145

ELECTRONIC

COMMUNICATIONS

in PROBABILITY

\title{
CONNECTED ALLOCATION TO POISSON POINTS IN $\mathbb{R}^{2}$
}

\author{
MAXIM KRIKUN \\ Institut Élie Cartan, Université Henri Poincaré, BP 239, 54506 VANDOEUVRE-LES-NANCY \\ CEDEX - France \\ email: krikun@iecn.u-nancy.fr
}

Submitted February 14 2007, accepted in final form April 202007

AMS 2000 Subject classification: 60D05

Keywords: Poisson process, Riemann map

Abstract

This note answers one question in [1], concerning the connected allocation for the Poisson process in $\mathbb{R}^{2}$. The proposed solution makes use of the Riemann map from the plane minus the minimal spanning forest of the Poisson point process to the halfplane. A picture of a numerically simulated example is included.

\section{The problem}

Let $X \subset \mathbb{R}^{d}$ be a discrete set. We call the elements of $X$ centers, the elements of $\mathbb{R}^{d}$ - sites, and we write $\mathcal{L}$ for the Lebesgue measure in $\mathbb{R}^{2}$. An allocation of $\mathbb{R}^{d}$ to $X$ with appetite $\alpha \in[0, \infty]$ is a measurable function $\psi: \mathbb{R}^{d} \rightarrow X \cup\{\infty, \Delta\}$ such that $\mathcal{L} \psi^{-1}(\xi) \leq \alpha$ for all $\xi \in X$ and $\mathcal{L} \psi^{-1}(\Delta)=0$. We call $\psi^{-1}(\xi)$ the territory of the center $\xi$. A center $\xi$ is sated if $\mathcal{L} \psi^{-1}(\xi)=\alpha$ and unsated otherwise. A site $x$ is claimed if $\psi(x) \in X$ and unclaimed if $\psi(x)=\infty$. The allocation is undefined at $x$ if $\psi(x)=\Delta$.

One particular question we're interested in is the following [1]: Is there a translation-equivariant allocation for the Poisson process of unit intensity in the critical two-dimensional case ( $d=2$ and $\alpha=1$ ), in which every territory is connected? The goal of the present note is to describe one such allocation. Note: for a far more general approach to this question in $d \geq 3$ see [3].

\section{Construction of a connected allocation}

Let $X$ be a realization of Poisson process (of intensity 1 ) in $\mathbb{R}^{2}$.

Let the minimal spanning forest $T$ be the union of edges $e=(x, y), x, y \in X$, such that there is no path from $x$ to $y$ in the complete graph on $X$ with all edges strictly shorter than $e$ (see [4] for other definitions and references on the subject).

For a 2-dimensional Poisson process Alexander [2] proved that $T$ is a.s. a tree with one topological end (a topological end in a graph is a class of equivalence of semi-infinite paths modulo finite 
symmetric difference). Thus the domain $D=\mathbb{R}^{2} \backslash T$ is simply connected, and by the Riemann mapping theorem can be mapped conformally to the upper half-plane $\mathbb{H}=\{z \in \mathbb{C}: \Im(z)>0\}$. Moreover, we can choose such a mapping $f$ which also sends infinity to infinity. (Note that any two such functions differ by a conformal automorphism of $\mathbb{H}$ which invariates infinity, i.e. by an affine mapping $z \rightarrow a z+b, a, b \in \mathbb{R}, a \neq 0)$.

The mapping $f$ cannot be extended unambiguously to $T$. Instead, consider the inverse mapping $g=f^{-1}$ from the open halfplane to $D$, then extend $g$ to the real axis by continuity, obtaining a surjection $\bar{g}: \overline{\mathbb{H}} \rightarrow \mathbb{R}^{2}$. Some points of $X$ may have more than one preimage under $\bar{g}$, more exactly, a point has as many preimages as it's degree in $T$. For those points we'll have to split their appetite between these preimages in some way, e.g. proportionally to the angle of the corresponding sector. Let $\left(x_{n}^{\prime}, \alpha_{n}^{\prime}\right)$ be the resulting points and appetites. In the following we call the countable set $\left\{\left(x_{n}^{\prime}, \alpha_{n}^{\prime}\right), n \in \mathbb{Z}\right\}$ the image of $X$ under $f$.

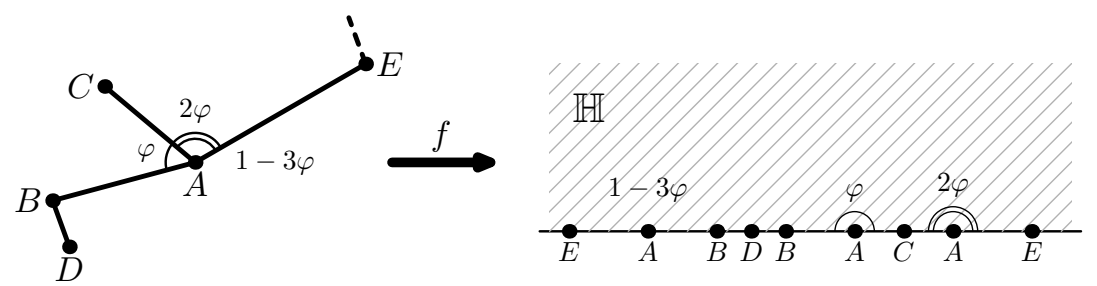

Figure 1: The image of $X$ under $f$; the unit appetite of the center $A$ is split into three unequal parts.

Now consider the following stable allocation procedure (see Appendix II for a formal definition):

- Each center starts growing a ball centered in it. All the balls grow simultaneously, at the same linear speed.

- Each center claims the sites captured by it's ball, unless the site was claimed earlier by some other center.

- Once the center becomes sated (the measure of it's territory reaches it's appetite), the ball stops growing.

Note that from our choice of function $f$ it follows that the set $\left\{x_{n}^{\prime}, n \in \mathbb{Z}\right\}$ is locally finite (i.e. has no accumulation point other than infinity), so the stable allocation procedure is well defined.

Applying this procedure to the image of $X$ under $f$ in the half-plane $\mathbb{H}$, with respect to the Euclidean metric in $\mathbb{H}$ and the image $\lambda$ of the Lebesgue measure $\mathcal{L}$ under $f$ yields certain allocation $\psi_{H}: \mathbb{H} \rightarrow X \cup\{\infty, \Delta\}$.

Lemma 2.1. The allocation $\psi_{H}$ thus constructed has connected territories and is invariant under affine transformations of $\mathbb{H}$.

Proof. The affine invariance follows immediately by construction.

To show that each center has connected territory, consider the following. Let $A$ be a center, necessarily located at the boundary of $\mathbb{H}$, and let $D_{A}=\psi_{H}^{-1}(A)$ be it's territory. Consider the ray $A A^{\prime}$, perpendicular to $\mathbb{R}$. If some point $x$ on $A A^{\prime}$ doesn't belong to $D_{A}$, that's because the center got sated before reaching $x$, so no further point on $A A^{\prime}$ belongs to $D_{A}$. Thus the intersection of $D_{A}$ with $A$ is a segment $A K$. 


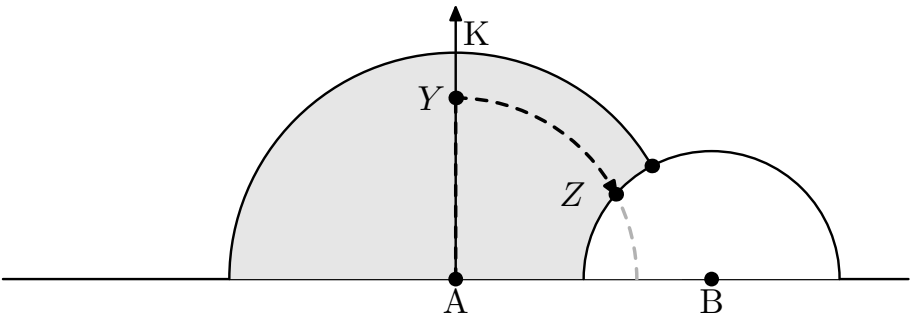

Figure 2: Every point of $D_{A}$ can be reached from $A$

Now consider an arc of a circle centered in $A$ and intersecting $A K$ in some point $Y$, and follow this arc to the right (or left) from $Y$. Once we meet a point $Z \notin D_{A}$, there must be a center $B$ such that $B Z \leq A Z$, so no further points on the arc belong to $D_{A}$. Thus every point of $D_{A}$ can be reached from $A$, and $D_{A}$ is connected.

Now apply the inverse map $f^{-1}$ to $\psi_{H}$. i.e. let $\psi=f^{-1} \psi_{H} f$. Clearly, $\psi$ is an allocation of $\mathbb{R}^{2}$ to $X$ with appetite $\alpha$, and from the previous lemma it doesn't depend on the choice of $f$.

Lemma 2.2. The allocation $\psi$ is translation-invariant, i.e. if $\tau: \mathbb{R}^{2} \rightarrow \mathbb{R}^{2}$ is a translation, and $\psi^{\prime}$ it the allocation or $\mathbb{R}^{2}$ to $\tau X$ constructed using the above procedure, then $\psi^{\prime}=\tau^{-1} \psi \tau$.

Proof. Let $X^{\prime}=\tau X$. Clearly, the minimal spanning forest is translation-invariant, i.e. $T^{\prime}:=$ $M S F\left(X^{\prime}\right)=\tau \cdot M S F(X)$. Now $f^{\prime}=f \tau^{-1}$ is a conformal function that maps $\mathbb{R}^{2} \backslash T^{\prime}$ to $\mathbb{H}$, and the image of $X^{\prime}$ under $f^{\prime}$ coincides with the image of $X$ under $f$, thus $\psi^{\prime}=f^{\prime-1} \psi_{H} f^{\prime}=$ $\tau^{-1} f^{-1} \psi_{H} f \tau=\tau^{-1} \psi \tau$.

Lemma 2.3. Under the allocation $\psi$ every center is sated a.s.

Proof. First, it follows from the Gale-Shapley algorithm (see Appendix II below), that no stable allocation may have both unclaimed sites and unsated centers. By ergodicity, the existence of unclaimed sites is a $0 / 1$ event; thus we may assume that $\psi_{H}$, and therefore $\psi$, have no unclaimed sites.

Now Lemma 16 in [1] states that for any translation-invariant allocation $\psi$ and any $r>0$

$$
\mathrm{P}\{|\psi(0)|<r\}=\mathrm{E}^{*} \mathcal{L}\left(\psi^{-1}(0) \cap B(0, r)\right),
$$

where $E^{*}$ denotes the expectation with respect to the Poisson process conditioned to have a center in 0 . Taking $r \rightarrow \infty$ yields

$$
\mathrm{P}\{0 \text { is claimed }\}=\mathrm{E}^{*} \mathcal{L} \psi^{-1}(0) .
$$

But we assumed the probability on the left to be equal to 1 , and $\mathcal{L} \psi^{-1}(0) \leq 1$ by construction (a center cannot allocate more than it's appetite), thus the lemma follows.

We summarize the above lemmas in the following

Theorem 1. The allocation $\psi$ thus constructed has connected territories, is translationinvariant, and every center is sated a.s. 


\section{Further questions}

Geometry of territories. Is it true that the every territory $\psi^{-1}(\xi)$ is bounded a.s.? The territories of $\psi$ are connected, but, a priori, may be not simply connected (more exactly, their closures may not be simply connected). Can the construction of $\psi$ be modified to assure this? Point process on $\mathbb{R}$. What can be told about the image of $X$ under the Riemann map, as a point process on $R$ ? Simulations suggest that this process should have extremely high variation of point density; even for small polygons of 30-40 points, the distances between the images of consecutive points may vary from $\approx 1$ to $\approx 10^{-16}$.

Fixing the Riemann map. The Riemann map $f$ is unique up to a conformal automorphism of $H, z \rightarrow a z+b$. Is it possible to pick (deterministically) one particular mapping from this class? If omitting the translation-invariance, one obvious way to do this is to pick a mapping $f$ that sends 0 to $i$. If doing this in translation-invariant manner, there is obviously no way to fix the shift $b$ (since this would imply the deterministic translation-invariant choice of single point from the Poisson process). But does there exist a way to pick the scale, i.e. fix the parameter $a$ ?

\section{References}

[1] Christopher Hoffman, Alexander E. Holroyd, and Yuval Peres. A stable marriage of Poisson and Lebesgue. Annals of Probability, 34:1241, 2006, math.PR/0505668. MR2257646

[2] Kenneth S. Alexander. Percolation and minimal spanning forests in infinite graphs. Annals of Probability, 23:87-104, 1995. MR1330762

[3] Sourav Chatterjee, Ron Peled, Yuval Peres, and Dan Romik. Gravitational allocation to Poisson points, 2006, math.PR/0611886.

[4] Russell Lyons, Yuval Peres, and Oded Schramm. Minimal spanning forests. Annals of Probability, 34:1665, math.PR/0412263. MR2271476

[5] Donald E. Marshall and Steffen Rohde. Convergence of the zipper algorithm for conformal mapping, 2006, math.CV/0605532. 


\section{Appendix I: An obligatory picture}

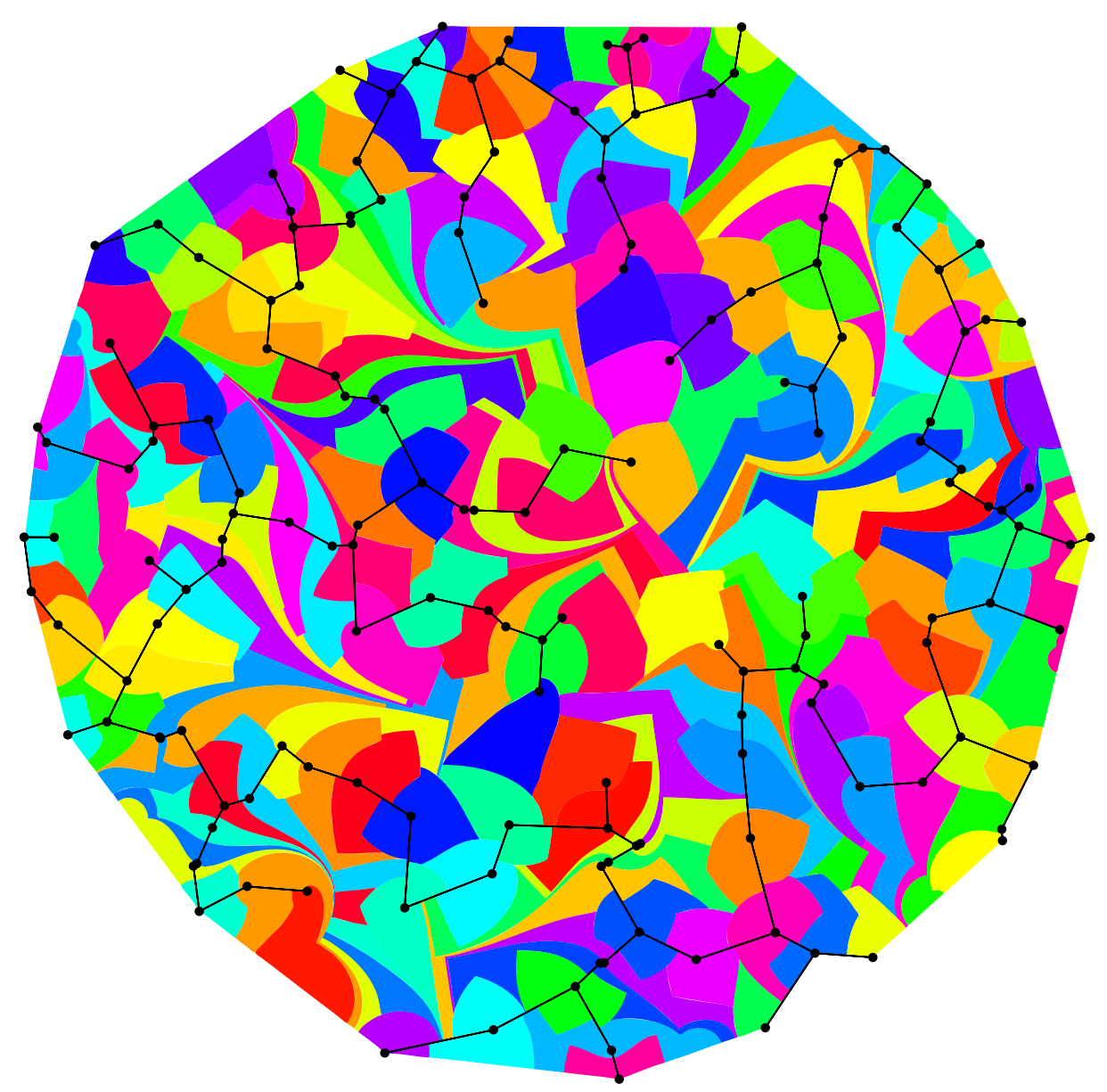

Figure 3: An approximation of allocation for 164 points in the unit circle

On the above picture we took 164 uniformly distributed points inside the unit circle, and mapped each component $K_{j}$ of their convex hull minus their MST to a region of $\mathbb{H}$, so that all the internal edges are mapped to $\mathbb{R}$. Then for each component we allocated it's whole area to the bounding corners, choosing the appetites proportional to the angular measure.

The mapping was approximated with a version of the geodesic algorithm, adapted for domains with non-Jordan boundary (or more exactly, for domains with boundary consisting of a Jordan curve plus a number of internal trees); for discussion of numeric algorithms for quasi-conformal mappings see e.g. [5] and references therein.

Since the ratio area/ $\{$ sum of appetites $\}$ varies in different components $K_{j}$, not all domains have exactly the same area; there are also some artifacts related to the limited numeric accuracy. Nevertheless, we expect the picture close to the center to be an accurate approximation of the construction described in this note. 


\section{Appendix II: site-optimal Gale-Shapley algorithm}

(The description of the G-S algorithm below is taken from [1] except for the last step, when we have to be more careful with the definition of $\psi$ on $W$ in order to assure the connectedness of the territories.)

Let $W$ be the set of all sites, equidistant from one or more centers. Since the set of centers is countable, $W$ has Lebesgue measure null.

We construct $\psi$ on $\mathbb{R}^{2} \backslash W$ by means of a sequence of stages, where stage $n, n=1,2,3, \ldots$ consists of two steps:

a) Each site $x \notin W$ applies to the closest center, which has not rejected $x$ at any earlier stage.

b) For each center $\xi$, let $A_{n}(\xi)$ be the set of sites which applied to $\xi$ on step a of stage $n$, and define the rejection radius as

$$
r_{n}(\xi)=\inf \left\{r: \mathcal{L}\left(A_{n}(\xi) \cap B(\xi, r)\right) \geq \alpha\right\},
$$

where the infimum over the empty set is taken to be $\infty$. Then $\xi$ shortlists all sites in $A_{n}(\xi) \cap B\left(\xi, r_{n}(\xi)\right)$, and rejects all sites in $A_{n}(\xi) \backslash B\left(\xi, r_{n}(\xi)\right)$.

Now either $x$ is rejected by every center (in order of increasing distance from $x$ ), or $x$ is shortlisted by $\xi$ for some center $\xi$ at some stage $n$. In the former case we put $\psi(x)=\infty$ (so $x$ is unclaimed), in the latter case we put $\psi(x)=\xi$.

Finally, for $x \in W$ put $\psi(x)=\xi$, if $\xi$ is the only center such that $x \in \partial \psi^{-1}(\xi)$, and put $\psi(x)=\Delta$ (undefined) otherwise. 